Sādhanā, Vol. 18, Part 6, December 1993, pp. 891-910. (C) Printed in India.

\title{
On the intricacies in the dynamic regulatory control of chaotic systems ${ }^{\dagger}$
}

\author{
JAYANTA K BANDYOPADHYAY ${ }^{1}$, V RAVI KUMAR ${ }^{1}$, \\ B D KULKARNI*1 and P B DESHPANDE ${ }^{2}$ \\ ${ }^{1}$ Chemical Engineering Division, National Chemical Laboratory, Pune \\ 411008 , India \\ ${ }^{2}$ Chemical Engineering Department, University of Louisville, Kentucky \\ 40292, USA
}

MS received 13 November 1992; revised 29 May 1993

\begin{abstract}
Issues related to the dynamic regulatory control of a chaotic process are discussed and illustrated using phenomenological descriptions of nonlinear systems from different disciplines. The controller design is based on internal model control principles with a capability of handling constant load and stochastic disturbances. Simulation studies assess the control performance and the studies reveal the intricate stability features of chaotic dynamics which need to be considered for regulatory control. The studies also suggest that a proper choice of the controller variable, leading to a suitable range in the values for the controller output, may be required.
\end{abstract}

Keywords. Nonlinear control; internal model control; control of chaos; chaotic dynamics.

\section{Introduction}

In recent years, a subject of importance that has emerged is the study of the observed dynamical chaos in many physical, chemical and biological systems (e.g., see Berry et al 1987, Grebogi et al 1987, Thompson \& Stewart 1989). It is generally accepted that chaos arises due to nonlinear process characteristics which allow a system to show extreme sensitivity to parameter values as well as initial and boundary conditions. Indeed, dramatic variations in the dynamic output behaviour can ensue for insignificant errors in specifying the initial state of the system. Comprehension of the behaviour of a chaotic system from both theoretical and practical points of view is considerably enhanced by the availability of a prototype mathematical model which may be obtained either from a priori mechanistic knowledge or from experimental data. Apart from other interesting system features, a study of the mathematical model can possibly indicate the advantages that may arise when a chaotic system operates in an identified parameter/phase space. This suggests that it would be useful to design control methods

\footnotetext{
${ }^{\dagger} \mathrm{NCL}$ commun. no. 5668; *For correspondence
} 
that would allow a practical system to operate within the framework of a chosen set of abjectives. Understandably, any control method for a chaotic system would require considerable rigor due to its inherent dynamic sensitivity features. In this article, therefore, we propose to discuss in some detail the special features which need to be surmounted for the dynamic control of chaotic systems. We shall highlight their role by attempting to control the chaos arising in seminal model examples from the engineering, physical and chemical sciences.

Several recent reviews and articles (Cutler \& Ramaker 1980; Richalet et al 1982; Isidori 1985; Kravaris \& Chung 1987; Lee \& Sullivan 1988; Kravaris \& Kantor 1990a , b; Kravaris \& Soroush 1990; Limqueco \& Kantor 1990; Bequette 1991; Gattu \& Zafiriou 1992) covering different perspectives in the design of nonlinear controllers are available. As seen in these articles, among the different techniques studied for controlling nonlinear systems, the method of internal model control (IMC) has developed considerably and controller designs based on linearized versions of the model (Garcia \& Morari 1982) to those that are totally nonlinear (Economou et al 1986) have been carried out. Loosely speaking, formulating the control strategy on these lines uses advanced levels of mathematics. Further, stability considerations for a controlled system have shown that the incorporation of a linear or nonlinear filter to impart robustness to the controller may be required (Henson \& Seborg 1991). Employing a different approach, an IMC controller design has been proposed which can regulate the dynamics of a nonlinear system at a desired stable stationary state (Kulkarni et al 1991). The inclusion of an adaptive mechanism for the model parameters in the procedure has found application in controlling a number of more difficult situations arising due to the process operating in multistationarity and unstable regions, presence of noise etc. (Ravi Kumar et al 1991; Bandyopadhyay et al 1993b; Shukla et al 1993).

Broadly stated, most internal model based nonlinear controllers use state variable feedback in some form or the other to compute the driving action in a properly chosen manipulable variable of the system. The monitoring of state variables dynamically is usually limited by the availability of measurement methods with the associated sampling time constraints, and can therefore pose real-time difficulties in achieving control of chaotic processes. In this respect control algorithms which do not use state feedback are better placed (Hubler 1989; Hubler \& Luscher 1989; Jackson 1990; Jackson \& Hubler 1990), although they tolerate mismatch in process-model parameter value specifications and modelling errors (inadequate model representation of the process) to a lesser extent. Also, disturbance rejection can be better handled by allowing for a state feedback mechanism in the control strategy. A control algorithm incorporating feedback may be more generally applicable than one without, and for this reason, a method with feedback for controlling chaos has been developed (Bandyopadhyay et al 1992). In this study the original framework of IMC was improvised so as to make it applicable to the requirements of controlling chaotic systems. The noteworthy feature of this method has been to additionally include a feedback mechanism that minimizes the deviations in the rates of change of the process variables with those of the desired dynamics. We shall, for the purpose of studying the various aspects involved in controlling chaotic systems, employ this method so as to facilitate comparison of results.

This paper is structured as follows. In subsequent sections we shall summarize the pertinent aspects of the control alogrithm in a general fashion and also discuss the issues related to controlling chaotic dynamics. We then present the results of simulation 
studies which bring out the system features on attempting control for three representative chaotic systems with different dynamical topologies highlighting these issues.

The first system to be considered is that which is used in engineering applications and very often as a generic model, for its simplicity in conceptualizing a number of physical-cum-chemical processes, i.e., the exothermic reaction in a continuous stirred tank reactor (see, Kahlert et al 1981, Jorgensen \& Aris 1983, Jorgensen et al 1984, Retzloff et al 1987). The second example is the well-studied idealized mathematical model of Lorenz (Lorenz 1963; Sparrow 1982; Guckenheimer \& Holmes 1983) describing the thermally driven fluid convection considered by Rayleigh. Simple chemical reactions with the minimum of nonlinearity have been proposed and analysed for their bifurcation structures and complex dynamics. A mechanism from this class is the autocatalator model (Peng et al 1990, 1991; Scott et al 1991) and we shall therefore study a possible controller law formulation for this system.

It may be worth noting that controlling chaos for situations when a mathematical model for the system is not available is also being actively pursued. Thus, in the instance when time series data are available, Ott $e t$ al (1990) have suggested a method for the regulatory control of a chaotic system by analysing the system properties for reduced dimensionality on a suitably chosen Poincaré section. Specifically, a proportional feedback controller has been designed which requires an initial standardization of the sensitivity characteristics of the system for small parameteric variations in a controlled variable. Applications of this method to important nonlinear systems are also available (Ditto et al 1990; Peng et al 1991; Roy et al 1992; Bandyopadhyay et al 1993a).

\section{Basic principles for the control strategy}

Our first assumption for controlling a chaotic process is that a reasonably accurate process mathematical model is known. Second, for the purpose of stating our control objectives we assume that controlling a chaotic process implies regulating the dynamics of the process on some chosen orbit (set orbit) embedded in the chaotic attractor. We may identify these orbits by experiment or by solving the process mathematical model such that the beginning and end points of the chosen orbit lie in the dense region of the chaotic attractor. Thus, suitable control action should facilitate moving the process state to the initial point of the set orbit on dynamically reaching the neighbourhood of its end point. The controlled process can then follow the set orbit dynamics in a periodic fashion. To help outline and discuss the control framework the block diagram in figure 1 shows the linkages between the process, its mathematical model and controller design. We shall develop the subject matter based on this diagram section-wise.

\subsection{Process description}

We begin by writing in a general fashion the process dynamics to be described by a set of ODE of the type,

$$
\mathrm{dx} / \mathrm{d} t=\mathbf{f}(\mathbf{x}, \mathbf{k})+u_{\mathbf{t}} \mathbf{f}^{\prime}(\mathbf{x}, \mathbf{k})+\mathbf{d},
$$

where $\mathbf{x}$ is an $N$-dimensional vector of process state variables for the system, $\mathbf{k}$ a set 


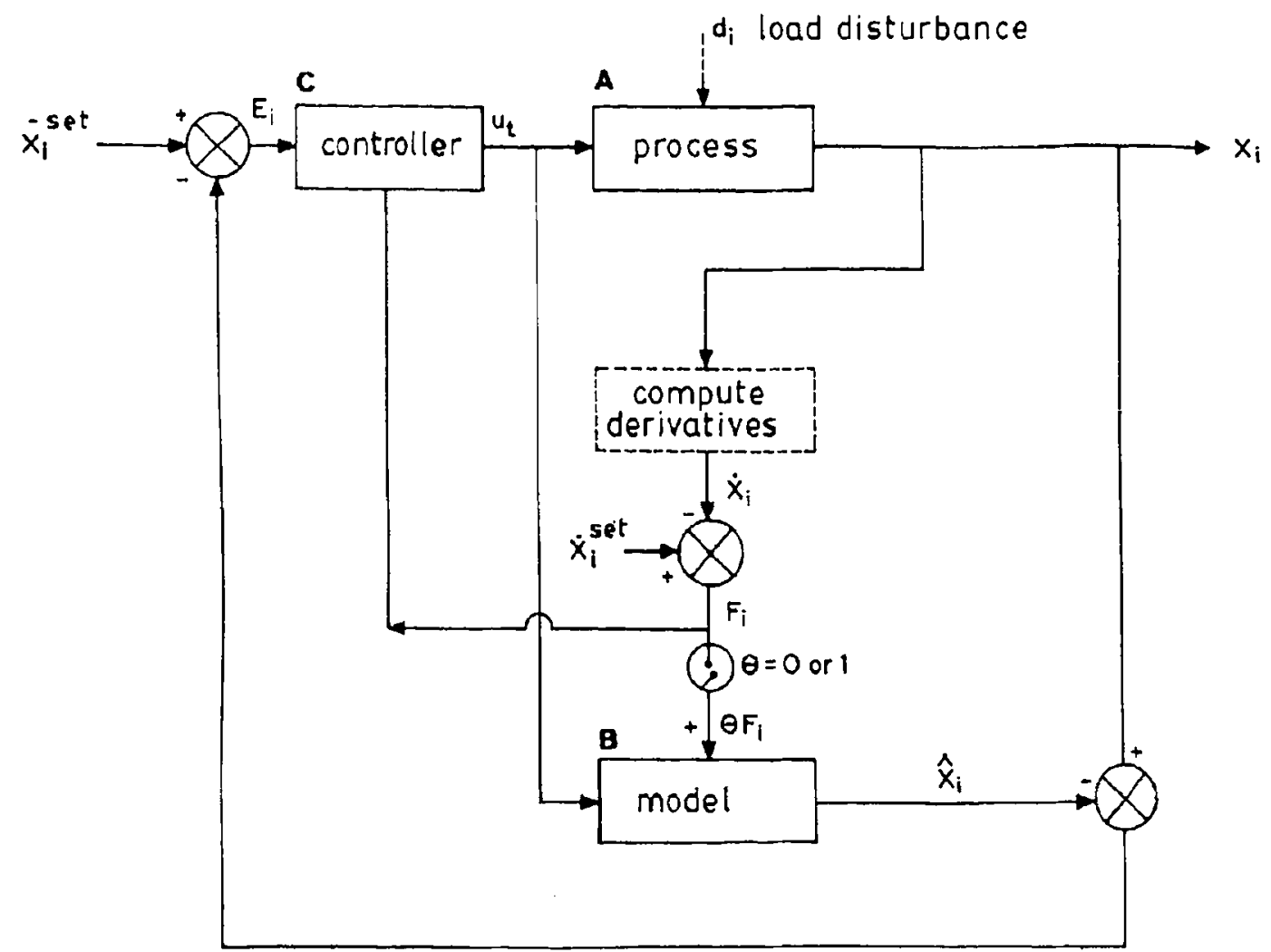

Figure 1. Schematic showing implementation of internal model control (IMC) for a chaotic process.

of process parameters and $u_{t}$ a manipulable control variable whose magnitude can be dynamically adjusted on the process by external means. The $f$ and $f^{\prime}$ represent nonlinear functions affecting the time evolution of the process. The second term on the RHS, $u_{f} \mathbf{f}^{\prime}$ indicates that the manipulable variable is of a separable form and can always be solved explicitly by algebraic manipulation and substitution. The vector d allows for stochastic or constant disturbances that the process may experience in time from various sources. Although we have in hand a mathematical model for the process, it may be emphasized at this point that the process equations in the form of (1) are used only for simulation purposes and in real-life applications the $\mathbf{x}$ will be the measured quantities of the state variables. The process has been depicted by Block $\mathrm{A}$ in figure 1.

\subsection{Model description}

Without any loss in generality we may write the set of equations to describe the mathematical model of the process in terms of the model state variables $\hat{\mathbf{x}}$ as

$$
\mathrm{d} \hat{\mathbf{x}} / \mathrm{d} t=\mathbf{f}(\hat{\mathbf{x}}, \hat{\mathbf{k}})+u_{t} \mathbf{f}^{\prime}(\hat{\mathbf{x}}, \hat{\mathbf{k}})+\hat{\mathbf{d}},
$$

where introducing the notation $\hat{\mathbf{k}}$ allows for setting parameter values of the model different from the true operating value in the process as specified in (1). A similar argument holds for the load disturbance vector $\hat{d}$, where for $\mathbf{d}$ in (1) with finite values for its elements, if $\hat{\mathbf{d}}$ is null then the model outputs $\hat{\mathbf{x}}$ do not consider the presence 
of the loads on the process. In figure 1, Block B denotes the available model description.

\section{2a Convergence considerations for chaotic dynamics}

We now draw attention to the non-intersecting property of the phase space trajectories of a deterministic process. This property allows the dynamics of the system at any given instant to be calculated using the model, provided the values of the state variables are known precisely. Sensitivities of nonchaotic systems to a lack of precision in inputs are much lower than the dynamics of chaotic systems which calls for a very high degree of precision in state variable measurements $\mathbf{x}$. In real-life, measurements of this order are not easily achievable. One way to give the mathematical model better information for calculating model outputs for control purposes is to consider including in the mathematical model, the effects of deviations in the evolutionary directions of the process trajectories from those of the set orbit. Thus, the $N$ local difference in the derivatives (say elements of a vector $\mathbf{F}$ ) that exists in the state variables between those of the process (measured to the best possible precision) and the desired set trajectory dynamically, may be defined as

$$
\mathbf{F}=\left(\mathrm{d} \mathbf{x}^{\mathrm{set}} / \mathrm{d} t\right)-(\mathrm{d} \mathbf{x} / \mathrm{d} t)
$$

and included in the model (2) so as to obtain a set of modified model equations having the form

$$
\mathrm{d} \hat{\mathbf{x}}_{m} / \mathrm{d} t=\mathrm{f}\left(\hat{\mathbf{x}}_{m}, \hat{\mathbf{k}}\right)+u_{i} \mathbf{f}^{\prime}\left(\hat{\mathbf{x}}_{m}, \hat{\mathbf{k}}\right)+\hat{\mathbf{d}}+\mathbf{F} .
$$

For clarity we have the nomenclature for the modified model variable outputs in (4) identified as $\hat{\mathbf{x}}_{m}$. Particularly, when the derivative values of the process trajectories $\mathrm{dx} / \mathrm{d} t$ match those of the set orbit $\mathrm{d} \mathbf{x}^{\text {set }} / \mathrm{d} t$ dynamically $F$ becomes a null vector, indicating a direct correspondence between the above modified model (4) and the known mathematical model of the process (2). Note that elements of $F$ are numerical values that may be supplied to the model at every integration step-size.

The dynamical systems we consider are dissipative and so the trajectories of the chaotic attractor converge onto a lower-dimensional manifold after the initial transients are over and continue to do so without the trajectories intersecting in space. This is achieved by the trajectory repeatedly going through a process of folding and unfolding. Thus, in general, there are regions, where small perturbations (say, in the control variable $u_{t}$ ) given to the process may die down, or others where the trajectories would diverge. The knowledge of the process mathematical model (2) may be used to identify the phase space points wherein control is likely to achieve the objective. A possible method for ascertaining the convergence properties in the neighbourhood of a point on the trajectory is to monitor the process evolution properties of suitably chosen states lying on an infinitesimal hyper-sphere surrounding the point. A convenient choice of the chosen states would be those with an orthonormal frame of reference. The evolution properties may be studied by linearizing the model (2) and integrating sets of these equations for appropriate initial conditions, corresponding to the chosen states on the hyper-sphere surrounding the true trajectory point. The procedure can, therefore, facilitate the monitoring in time of the growth of the hyper-radii as integration proceeds. A decrease (increase) in the radii from those of the hyper-sphere in each (any) of the orthogonal directions would indicate 
that the system occupies a phase space point wherein the effects of perturbation would die down (grow). In essence, the method parallels the principles involved in calculating the Lyapunov exponents used for characterizing the presence of chaos in a system and we shall next discuss the physical significance of the exponents to bring about the connection.

\section{2b Including convergence considerations in the model by a switch $(\theta)$}

For an $N$-dimensional system following (2), the $N$-Lyapunov exponents quantify the sensitivity of system dynamics to its initial conditions and also indicate the degree to which nearby phase space trajectories diverge (Guckenheimer \& Holmes 1983; Bai-Lin 1984). Chaotic behaviour is exemplified by the presence of at least one positive Lyapunov exponent and a zero exponent. The existence of more than one positive Lyapunov exponent shows a hyper-chaotic system and arises due to orbits diverging in multiple directions in phase space. Limit cycle or more complex multi-peak periodic situations have an associated zero valued exponent with all others negative while stable dynamics have all negative exponents. The evaluation of the Lyapunov exponents from a known set of differential equations can be carried out by observing the evolution of small perturbations to the system orbit during its time evolution. Thus, if $J(\hat{\mathbf{x}}[t], \hat{\mathbf{k}}, \hat{\mathbf{d}})$ is the $N \times N$ Jacobian matrix evaluated along a dynamic system trajectory whose states are represented by the vector $\hat{\mathbf{x}}[t]$, then a perturbation $\delta \hat{\mathbf{x}}$, to the trajectory satisfies, the linear relationship

$$
\delta \hat{\mathbf{x}}[t+\Delta t]=J(\hat{\mathbf{x}}[t], \hat{\mathbf{k}}, \hat{\mathbf{d}}) \delta \hat{\mathbf{x}}[t]
$$

The divergence of nearby orbits can be studied by monitoring the growth rate of the infinitesimal $N$-dimensional hyper-sphere constructed about the initial state of the system trajectory by specifying a set of $N$ orthonormal vectors $\left(\alpha_{k}[t], k=1,2, \ldots N\right)$ around this state. Solution of the system equations with the $N$ sets of linearized equations of motion [obtained in the form of (5)] yields the growth rate of these vectors in time. The hyper-sphere is seen to deform into a hyper-ellipse and the multiplicative ergodic theorem (Osledec 1968) states that the characteristic radii of this hyper-ellipse converge in time. The Lyapunov exponents are related to the converged value of these radii. Let the norm $\left\|\alpha_{k}(\infty)\right\|, k=1,2, \ldots, N$ be the metric quantity measuring the magnitude of these radii vectors. The $N$ Lyapunov exponents $\left(\dot{\lambda}_{k}, k=1\right.$, $2, \ldots, N)$, can be calculated as

$$
\hat{\lambda}_{k}=\lim _{t \rightarrow \infty}(1 / t) \ln \left[\left\|\alpha_{k}[\infty]\right\|\right] /\left[\left\|\alpha_{k}[0]\right\|\right], \quad(k=1,2, \ldots, N),
$$

where if an orthonormal frame of reference is chosen, then, $\left\|\alpha_{k}(0)\right\|=1, k=1,2, \ldots, N$. To aid the numerical solution of the system (5) employing a time interval $\Delta t$, the discretized version of $(6)$ may be written as

$$
\lambda_{k}[t]=\left\{\sum_{1}^{r} \ln \left(\left\|\alpha_{k}[\infty]\right\| /\left\|\alpha_{k}[0]\right\|\right)\right\} /(r \Delta t), \quad(k=1,2, \ldots, N)
$$

where as $t \rightarrow \infty$ (7) converges to the Lyapunov exponents $\lambda_{k}$. Repeated applications of (5) can, however, lead to an ill-conditioned nature of the matrix $J(\hat{\mathbf{x}}[t], \hat{\mathbf{k}}, \hat{\mathbf{d}})$. Moreover, due to the finite precision normally used in calculations we observe that 
each of the vectors $x_{k}[t], k=1,2, \ldots, N$ tends to align itself with the local direction of most rapid growth and effectively all the $\alpha_{k}$ converge to the largest Lyapunov exponent. Ortho-renormalizing the $\alpha_{k}[t], k=1,2, \ldots, N$ 's at frequent intervals can circumvent these problems and yield reasonable estimates of the exponents in decreasing order.

It may be seen that evaluating the Lyapunov exponents involves a process of averaging the dynamical properties of the system trajectories in time. At every time step each contribution to the average portrays the local nature of the dynamics, in the sense that they provide a ready inference on whether the hyper-ellipse radii are shrinking or expanding. Therefore, to identify-the regions where control may be implemented we use the conservative condition of ensuring that the linear dynamics for points on the orthonormal frame of reference shrink towards the true system trajectory, i.e. we check to see if the values of

$$
\ln \left[\left\|\alpha_{k}(r \Delta t)\right\|\right] /\left[\left\|\alpha_{k}[0]\right\|\right]<0,
$$

for $k=1,2, \ldots, N$. For convenience let us assume that if the criterion in (8) is satisfied (or not satisfied) a switch $\theta$ takes on a value $\theta=1$ (or $\theta=0$ ).

Based on the above discussion it becomes clear that before obtaining modified model outputs $\mathbf{x}_{m}$, via (4), dynamically, it is necessary to check whether the process occupies a point in a convergent phase space. This step is clearly indicated in figure 1. Testing for the convergence may be carried out by evaluating the lhs of (8) and suggests that $F$ values be included in (4) only in such instances. Thus the modified model (4) may be written including the role of the switch $\theta$ as

$$
\mathrm{d} \hat{\mathbf{x}}_{m} / \mathrm{d} t=\mathbf{f}\left(\hat{\mathbf{x}}_{m}, \hat{\mathbf{k}}\right)+u_{t} \mathbf{f}^{\prime}\left(\hat{\mathbf{x}}_{m}, \hat{\mathbf{k}}\right)+\hat{\mathbf{d}}+\theta \mathbf{F} .
$$

\subsection{Controller design}

We shall now indicate the IMC methodology for designing an equation which calculates the controller outputs $u_{t}$ needed to direct process trajectories towards a desired orbit. As stated earlier (4) may be rearranged to obtain an explicit calculation for the manipulable variable in a function $g$, i.e.,

$$
u_{\mathrm{r}}=g\left(\mathrm{~d} \hat{\mathbf{x}}_{m} / \mathrm{d} t, \hat{\mathbf{x}}_{m}, \hat{\mathbf{k}}, \hat{\mathbf{d}}, \mathbf{F}\right) \text {. }
$$

Choosing a reference vector $\mathbf{x}_{s}$ whose $N$ elements describe a convenient reference state, e.g., the process condition at $t=0$ or those of a stationary solution of the system, we define instantaneous set points in deviation variable form as $\overline{\mathbf{x}}^{\text {set }}=\mathbf{x}^{\text {set }}-\mathbf{x}_{s}$. We can then dynamically obtain values of the output $u_{z}$ under controlled conditions as

$$
u_{t}=g\left(\mathbf{E}^{\prime}, \mathbf{E}, \mathbf{x}_{s}, \hat{\mathbf{k}}, \hat{\mathbf{d}}, \mathbf{F}\right)
$$

where the functional dependencies involving the variables in (10) are replaced by error signals $\mathbf{E}$. The error signal vector $\mathbf{E}$ is defined as $\mathbf{E}=\overline{\mathbf{x}}^{\text {set }}-\left(\mathbf{x}-\hat{\mathbf{x}}_{m}\right)$ and relate the set point $\left(\overline{\mathbf{x}}^{\text {set }}\right)$, the measured process outputs $(\mathbf{x})$, and the model outputs $\left(\hat{\mathbf{x}}_{m}\right)$. The $\mathbf{E}^{\prime}$ are the derivatives of the error signal vector $\mathbf{E}$. Thus, (11) is the desired expression for the controller action and, as may be noted, receives as an input, the correction terms $\mathbf{F}$ at all $t$. This is because $\mathbf{F}$ measures the derivative deviations between the set and the actual trajectory and provides the corrections necessary for 
appropriately orienting the trajectories. In convergent regions the controller outputs are additionally influenced by the modified model output $\hat{\mathbf{x}}_{m}$ [since the switch $\theta$ takes a value of unity in (9)] via the error signals $\mathbf{E}$. For a system mathematical model, the functional form for $g$ in (11) has to be suitably derived so that controller outputs may be calculated dynamically. Block $\mathrm{C}$ in figure 1 denotes the controller design arrived at in the form of (11). It may be noted that as a first step, in the present analysis derivatives in the error measurements are employed, although increased robustness may be achieved by incorporating filters of the Kalman type.

In the subsequent section, for the chaotic systems under study, we use appropriately derived forms for the controller block and discuss the obtained control performance via simulations.

\section{Results and discussion}

\subsection{Example 1. The control of a chaotic nonisothermal cont inuous stirred tank reactor}

We shall formulate the control strategy for a jacketed continuous stirred tank reactor (CSTR) in which an irreversible consecutive first-order endo-exothermic reaction $\mathrm{A} \rightarrow$ $\mathrm{B} \rightarrow \mathrm{C}$ takes place. The system has been analysed (Kahlert et al 1981; Bandyopadhyay et al 1992) and shows a sequence of period doubling bifurcations of a limit cycle to chaos for realistic parameter values. The dimensionless equations describing this system consist of mass and energy balances with provision for controlling the system by regulating the coolant temperature and are given by

$$
\begin{aligned}
& \mathrm{d} x_{1} / \mathrm{d} t=1-x_{1}-\mathrm{Dax} x_{1} \exp \left[x_{3} /\left(1+\varepsilon x_{3}\right)\right]-d_{2}, \\
& \mathrm{~d} x_{2} / \mathrm{d} t=-x_{2}+\operatorname{Dax} x_{1} \exp \left[x_{3} /\left(1+\varepsilon x_{3}\right)\right]-\operatorname{DaS} x_{2} \exp \left[\kappa x_{3} /\left(1+\varepsilon x_{3}\right)\right]-d_{3}, \\
& \mathrm{~d} x_{3} / \mathrm{d} t=-x_{3}+\operatorname{Da} B x_{1} \exp \left[x_{3} /\left(1+\varepsilon x_{3}\right)\right]-\operatorname{Da} B \alpha S x_{2} \exp \left[\kappa x_{3} /\left(1+\varepsilon x_{3}\right)\right] \\
& \quad-\beta\left(x_{3}-x_{3 c}\right)+\beta u_{t}+d_{1} .
\end{aligned}
$$

Here, $x_{1}, x_{2}$ represent dimensionless concentrations of species $A$ and $B$, while $x_{3}$ denotes the dimensionless temperature. The other system parameters are dimensionless and relate to: $B$, the adiabatic temperature rise; $\mathrm{Da}$, the Dämkohler number; $S$, ratio of the rate constants for the series reaction; $x_{3 c}$, a reference value for the dimensionless coolant temperature; $\alpha$, ratio of heat effects for the series reaction; $\beta$, the heat transfer coefficient; $\varepsilon$, dimensionless activation energy; and $\kappa$, ratio of activation energies for the series reaction. The term $u_{t}$ represents a deviation in the coolant temperature in the jacket from $x_{3 c}$ and may be conveniently chosen as the manipulated control variable. The terms $d_{1}, d_{2}, d_{3}$ are added to take account of load disturbances which the process may experience while on stream.

To distinguish the process from the mathematical model used in the IMC structure a modified model version of (12) as discussed earlier is now introduced with the variables $x_{i}$ replaced by $\hat{x}_{i, m}$ i.e.,

$$
\begin{aligned}
\mathrm{d} \hat{x}_{1, m} / \mathrm{d} t=1-\hat{x}_{1, m}-\operatorname{Dax} \hat{x}_{1, m} \exp \left[\hat{x}_{3, m} /\left(1+\varepsilon \hat{x}_{3, m}\right)\right]-\hat{d}_{2}+\theta F_{1}, \\
\mathrm{~d} \hat{x}_{2, m} / \mathrm{d} t=-\hat{x}_{2, m}+\operatorname{Dax} \hat{x}_{1, m} \exp \left[\hat{x}_{3, m} /\left(1+\varepsilon \hat{x}_{3, m}\right)\right] \\
\quad-\operatorname{DaS} \hat{x}_{2, m} \exp \left[\kappa \hat{x}_{3, m} /\left(1+\varepsilon \hat{x}_{3, m}\right)\right]-\hat{d}_{3}+\theta F_{2},
\end{aligned}
$$




$$
\begin{aligned}
\mathrm{d} \hat{x}_{3, m} / \mathrm{d} t=- & \hat{x}_{3, m}+\operatorname{DaB} \hat{x}_{1, m} \exp \left[\hat{x}_{3, m} /\left(1+\varepsilon \hat{x}_{3, m}\right)\right] \\
& -\operatorname{DaSB\alpha } \hat{x}_{2, m} \exp \left[\kappa \hat{x}_{3, m} /\left(1+\varepsilon \hat{x}_{3, m}\right)\right] \\
& -\beta\left(\hat{x}_{3, m}-\hat{x}_{3 c}\right)+\beta u_{t}+\hat{d}_{1}+\theta F_{3} .
\end{aligned}
$$

We shall now obtain the controller design equation for the process under study. The first step is to algebraically manipulate the modified model equation set (13) with $\theta=1$ so as to obtain an explicit functional form for $u_{t}$ in terms of the other system variables and parameters [i.e., in the form of (10)]. On doing so, we get

$$
\begin{aligned}
u_{i}=(1 / \beta)\{ & \dot{x}_{3, m}+x_{3, m}-B\left(1-x_{1, m}-\hat{d}_{2, m}+F_{1}-\dot{x}_{1, m}\right) \\
& +B \alpha\left(-\dot{x}_{2, m}-x_{2, m}+1-x_{1, m}-\hat{d}_{2}-\dot{x}_{1, m}-\hat{d}_{3}+F_{1}+F_{2}\right) \\
& \left.+\beta\left(x_{3, m}-x_{3 c}\right)-\hat{d}_{1}-F_{3}\right\} .
\end{aligned}
$$

Let us now define the following deviation variable $\bar{x}_{i}^{\text {set }}=x_{i}^{\text {set }}-x_{i s}$, and the error signals as $E_{i}=\bar{x}_{i}^{\text {set }}-\left(x_{i}-\hat{x}_{i, m}\right)$ with the respective derivatives $E_{i}^{\prime}=\mathrm{d} E_{i} / \mathrm{d} t$. As seen from the definition, the $E_{i}$ signals relate the set points $\left(x_{i}^{\text {set }}\right)$, the measured process outputs $\left(x_{i}\right)$ and the model outputs $\left(\hat{x}_{i, m}\right)$. Following the IMC methodology (see Kulkarni et al 1991) we may replace $x_{i, m}$ with $\left(E_{i}+x_{i s}\right)$ and $\dot{x}_{i, m}$ with $E_{i}^{\prime}$ in (14) to yield the final form of the controller equation

$$
\begin{aligned}
u_{t}=(1 / \beta)\left\{E_{3}^{\prime}\right. & +\left(E_{3}+x_{3 s}\right)-B\left[1-\left(E_{1}+x_{1 s}\right)-\hat{d}_{2}+F_{1}-E_{1}^{\prime}\right] \\
& +B \alpha\left[1-\left(E_{2}+x_{2 s}\right)-\left(E_{1}+x_{1 s}^{\prime}\right)-\hat{d}_{2}-\hat{d}_{3}+F_{1}+F_{2}-E_{1}^{\prime}-E_{2}^{\prime}\right] \\
& \left.+\beta\left[\left(E_{3}+x_{3 s}\right)-x_{3 c}\right]-\hat{d}_{1}-F_{3}\right\} .
\end{aligned}
$$

Dynamic solutions to the coupled set of differential equations (12) simulating the process, and the modified model (13) were obtained by using the IMSL gear package. The outputs from the process and the model, at increments of dimensionless time $\Delta t=0.0001$, were used to evaluate the error signals $E_{l}$ and $E_{i}^{\prime}$ by finite differences. These signals were then passed to the controller block for evaluating the controller output by (15). Each dynamically evaluated controller output $u_{t}$ was implemented on the process and simultaneously updated in the modified model prior to further time integration. To implement the derivative corrective action $F_{i}$ in (13), the validity of the criteria (8) (and thus the value for the switch $\theta$ ) was dynamically evaluated by solving simultaneously sets of linearized model equations (5) for an orthonormal frame of reference.

The process parameter values for chaotic system operation have been identified as $\mathrm{Da}=0.26, \varepsilon=0, S=0.5, \kappa=1, B=57.77, \alpha=0.426, x_{3 \mathrm{c}}=0$ and $\beta=7.99999$ and adapted from Kahlert et al (1981). The Lyapunov exponents calculated by (7) for the process operating in this parameter space were characterized as $(+, 0,-)$ confirming the presence of chaos (using the procedure of Shimada \& Nagashima 1979).

Figure 2a shows a two-dimensional projection of the dynamics of the chaotic attractor without load disturbances of any kind introduced. Choosing a dense region of this attractor a suitable set orbit was identified on which regulatory control of the system would be the desired goal (figure $2 b$ ). For control purposes it was assumed that the initial difference between the process outputs $\left(x_{i}\right)$ and set values $\left(x_{i}^{\text {sat }}\right)$ anywhere upto $5 \%$ could be tolerated before initiating the control action. Further, it was also ensured that these points $\left(x_{i}\right.$ and $\left.x_{i}^{\text {set }}\right)$ lie in the convergent phase space before initiating 

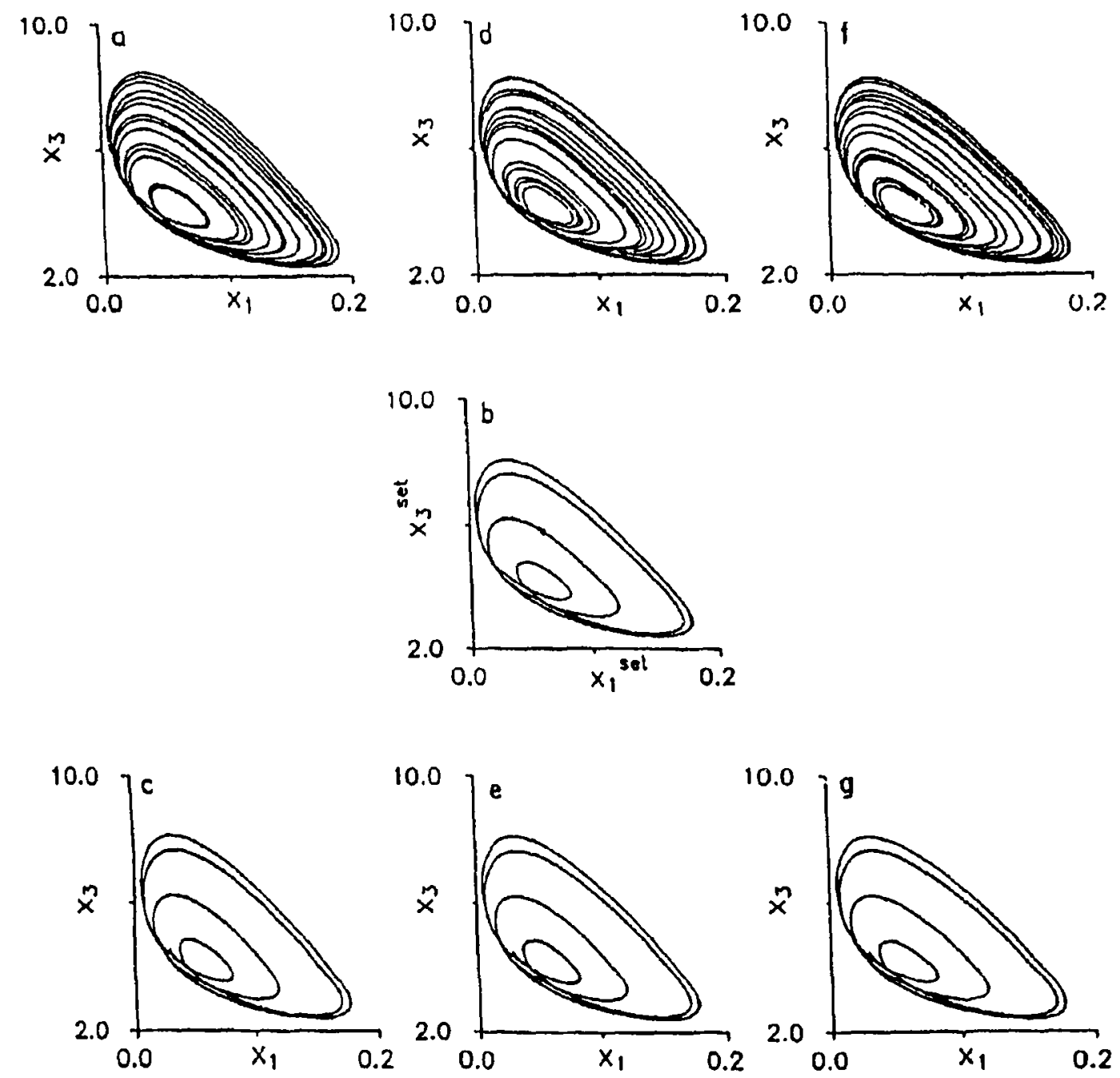

Figure 2. $x_{1}$ vs $x_{3}$ phase plane plots for the nonisothermal CSTR. Open loop, set and controlled dynamics in the absence ((a), (b), (c)) and in the presence ((d), (b), (e)) of a constant load disturbance; and in the presence of stochastic disturbances ((f), (b), (g)) respectively.
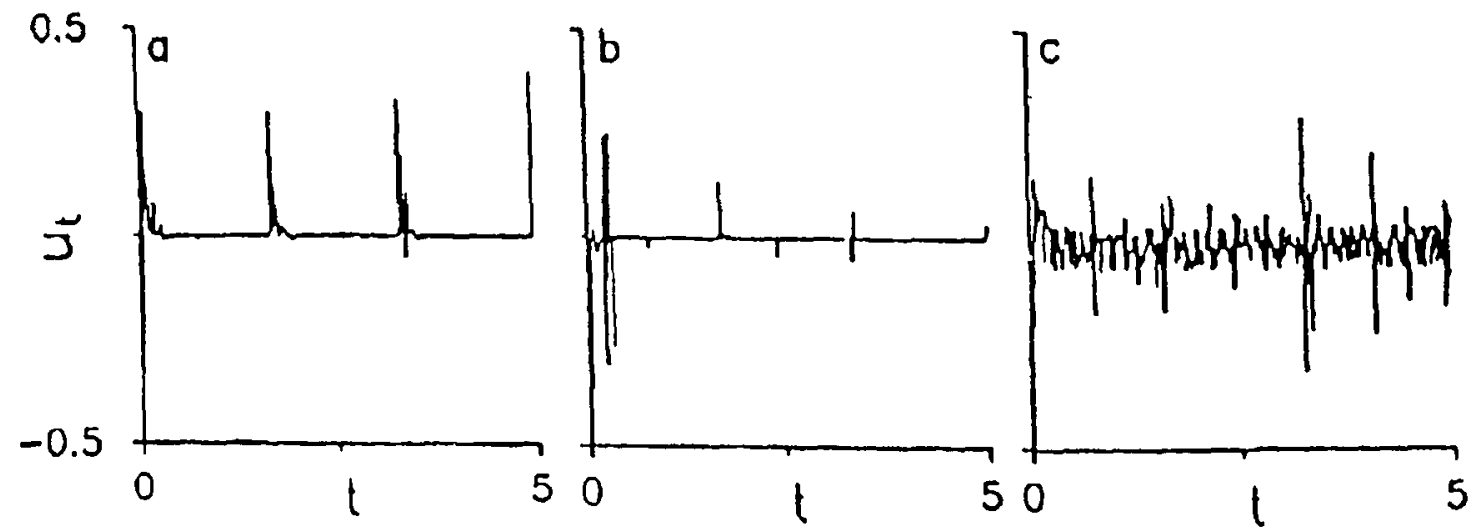

Figure 3. Controller action $\left(u_{t}\right)$ in time for the cases of regulatory control presented in figure 1. In the absence (a) and the presence (b) of a constant load disturbance; (c) in the presence of stochastic disturbances. 
the control action. The results obtained on simulation, with control implemented are shown in figure 2c. Clearly, the system dynamics shows that adequate control is achieved and the dynamically implemented controller outputs are shown in figure 3a.

It is of interest to re-examine the above problem in the presence of load disturbance that may occur in the process while on stream. The open loop process dynamics for a simple case when $d_{1}=0 \cdot 1, d_{2}=d_{3}=0$, is seen in figure $2 \mathrm{~d}$. We wish to control this problem with the constraint that the model is unaware of this disturbance $\left(\hat{d}_{i}=0.0\right)$. Thus, figure $2 \mathrm{e}$ shows the phase-space representation of the controlled process and figure $3 \mathrm{~b}$ the $u_{t}$ profile that is required to accomplish the dynamics of the desired orbit (i.e. figure $2 \mathrm{~b}$ ).

More frequently, the process may be subjected to stochastic load disturbances with the model being unaware of their presence. We have therefore simulated a situation where $d_{1}$ is of random character with a Gaussian distribution having a mean of 0.1 and variance of 0.25 . In the presence of noise the natural dynamics of the process (figure $2 \mathrm{f}$ ) is affected when compared to the corresponding open-loop process without any disturbance (figure 2a). Once again the calculated controller outputs (figure 3c) control the process which now follows the desired set dynamics (see figure $2 \mathrm{~g}$ ). Clearly, the capability to handle constant and stochastic load disturbances indicates controller robustness for the system.

\subsection{Example 2: Control of the Lorenz chaotic attractor}

We shall now formulate the control strategy for the seminal example of chaotic flow that arises from solving the hydrodynamic equations in a truncated form (Lorenz 1963). The nonlinear features of the Lorenz model have been reviewed in Sparrow (1982) and Guckenheimer \& Holmes (1983). The equation set describing the model is

$$
\begin{aligned}
& \mathrm{d} x_{1} / \mathrm{d} t=-\sigma\left(x_{1}-x_{2}\right)-d_{2}, \\
& \mathrm{~d} x_{2} / \mathrm{d} t=-x_{1} x_{3}-\left(\mathrm{r}+u_{t}\right) x_{3}-x_{2}-d_{3}, \\
& \mathrm{~d} x_{3} / \mathrm{d} t=x_{1} x_{2}-b x_{3}+d_{1} .
\end{aligned}
$$

Here, $x_{1}$ represents the amplitude of convection motion, $x_{2}$ the temperature difference between ascending and descending currents and $x_{3}$ the distortion of the vertical temperature profile from linearity. The other dimensionless parameters are $\sigma$ the Prandtl number, $r$ the normalized Rayleigh number and $b$ a geometry factor. The manipulable control variable $u_{t}$ is related to Rayleigh number. As in example 1 , the terms $d_{1}, d_{2}, d_{3}$ have been additionally added to take into account any load disturbances that the system may experience.

The modified mathematical model equations may now be written as:

$$
\begin{aligned}
& \mathrm{d} \hat{x}_{1, m} / \mathrm{d} t=-\sigma\left(\hat{x}_{1, m}-\hat{x}_{2, m}\right)-\hat{d}_{2}+F_{1}, \\
& \mathrm{~d} \hat{x}_{2, m} / \mathrm{d} t=-\hat{x}_{1, m} \hat{x}_{3, m}-\left(\mathrm{r}+u_{t}\right) \hat{x}_{3, m}-\hat{x}_{2, m}-\hat{d}_{3}+F_{2}, \\
& \mathrm{~d} \hat{x}_{3, m} / \mathrm{d} t=\hat{x}_{1, m} \hat{x}_{2, m}-b \hat{x}_{3, m}+\hat{d}_{1}+F_{3} .
\end{aligned}
$$

Following the procedure, (17) can be algebraically simplified assuming $\theta=1$ to obtain an explicit expression for $u_{t}$. Substitution of the error signals and derivatives in this expression gives a controller law for the system as,

$$
u_{1}=\left[E_{2}^{\prime}+\left(E_{2}+x_{2 s}\right)-F_{2} /\left(E_{1}+x_{1 s}\right)+\left(E_{3}+x_{3 s}\right)\right]-\mathrm{r} .
$$



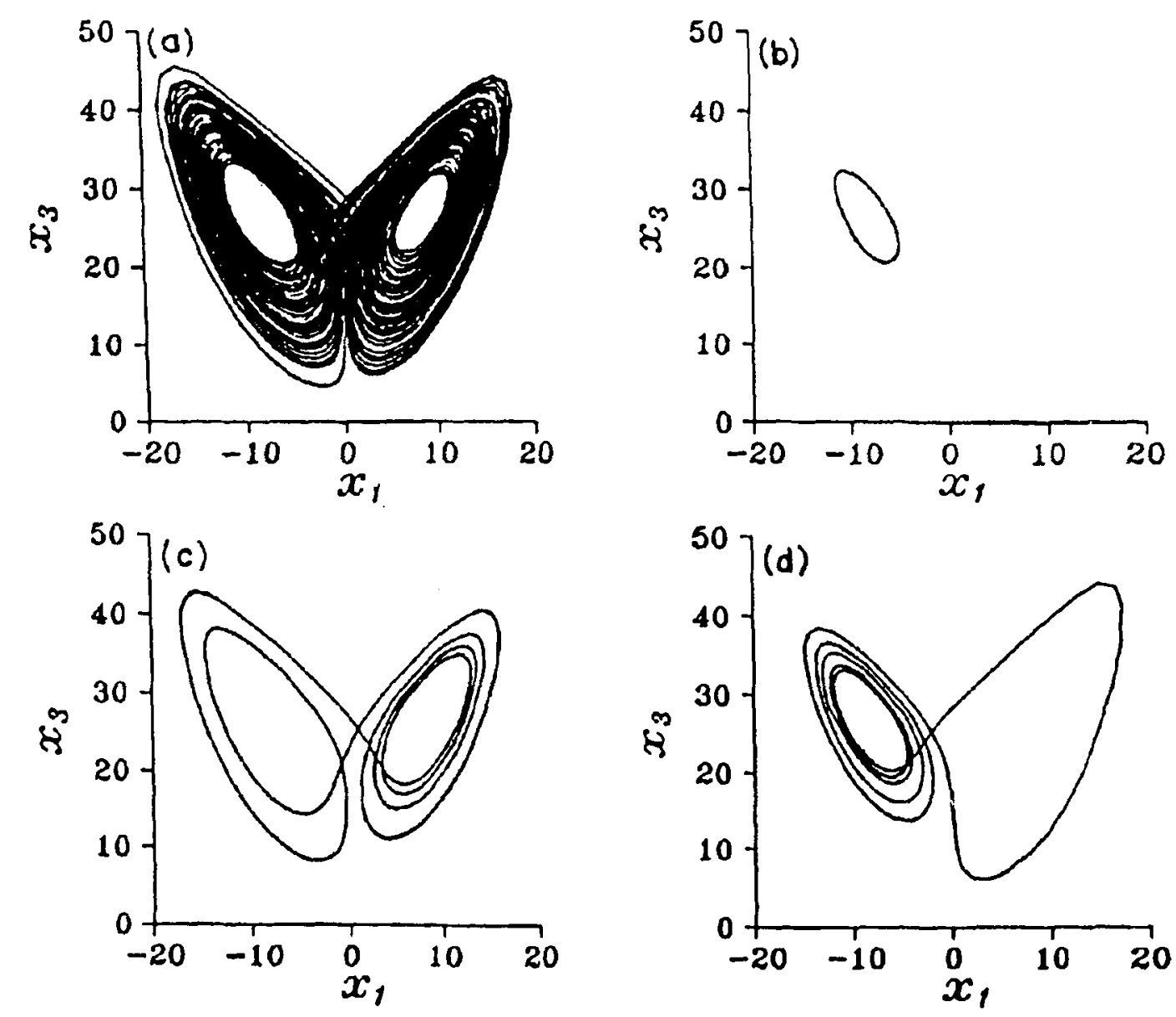

Figure 4. $x_{1}$ vs $x_{3}$ phase plane plots for the Lorenz model-open loop (a) and set orbits chosen for control studies $((\mathbf{b}, \mathbf{c}, \mathbf{d}))$.

We shall now present some representative results obtained on attempting control of this system under chaotic conditions. The process parameter values required for chaotic operation are: $\sigma=10.0, b=2.6667$ and $r=28.0$. Figure $4 a$ shows the phase plane plot $\left(x_{1}\right.$ vs $\left.x_{3}\right)$ of the chaotic attractor. The Lorenz model being highly oriented (i.e., non-equivalent left and right-handed trajectories exist) the choice of the conditions for initiating control can occur frequently and also give rise to topologically different set orbits. Among the various different possible configurations some typical set orbits are shown in figures $4 \mathrm{~b}, \mathrm{c} \& \mathrm{~d}$. Note that the trajectories at the beginning and the end have been closed to make the set trajectory into an orbit.

Figure $4 \mathrm{~b}$ which is similar to a period-1 orbit remains completely in the left hand quadrant and the corresponding time profiles are shown in figures $5 \mathrm{a}, \mathrm{b} \& \mathrm{c}$ for the variables $x_{1}, x_{2}$, and $x_{3}$. For initiating control, conditions similar to those in example 1 were imposed. Figures $5 d$, e \& f show the controlled trajectories in the variables and figure $5 \mathrm{~g}$ shows the manipulated variable movement. Clearly the set and process trajectories are identical and suggest effective chaos control.

Set trajectories in figures $4 c \& d$ have contributions from both the left and right half planes of the Lorenz attractor. The desired time profiles on control in $x_{1}$ corresponding to the two cases are shown in figures $6 \mathrm{a}$ and $7 \mathrm{a}$. The profiles in both the cases show them to be complex multi-peak periodic. The respective controlled profiles 


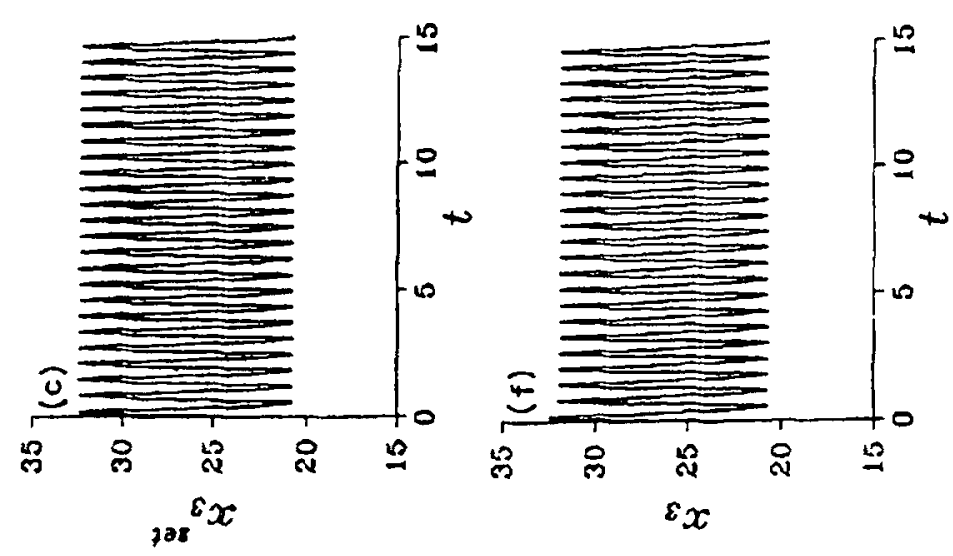

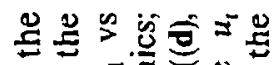

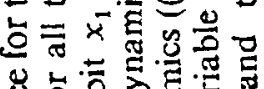

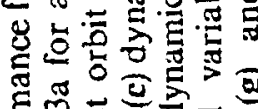

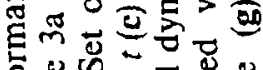

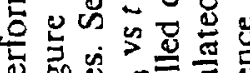

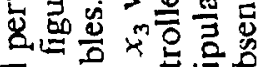

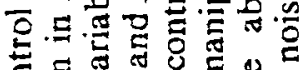

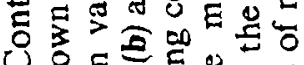

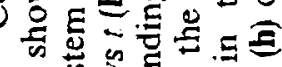

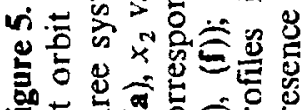

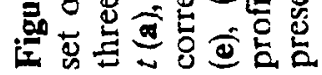
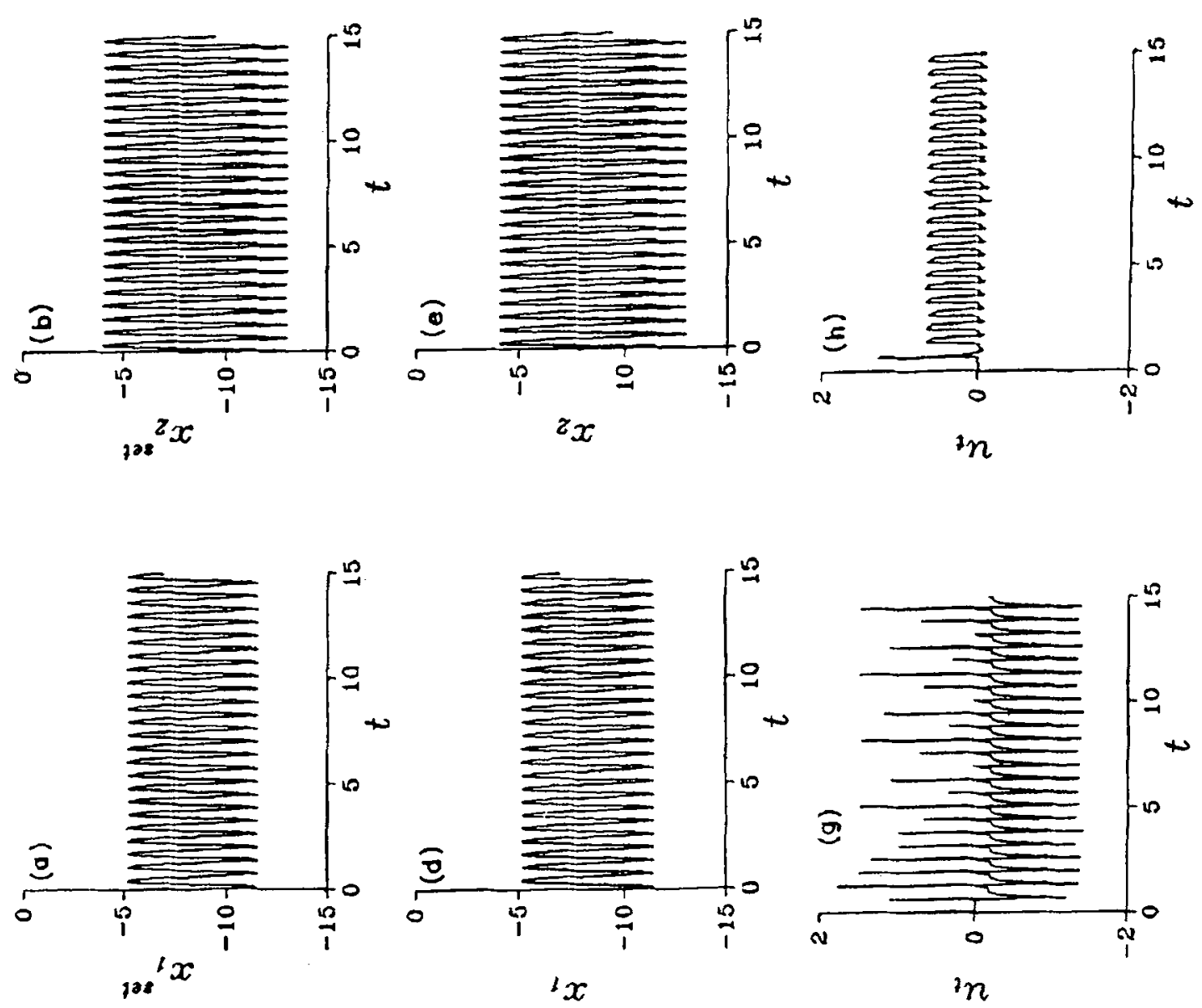

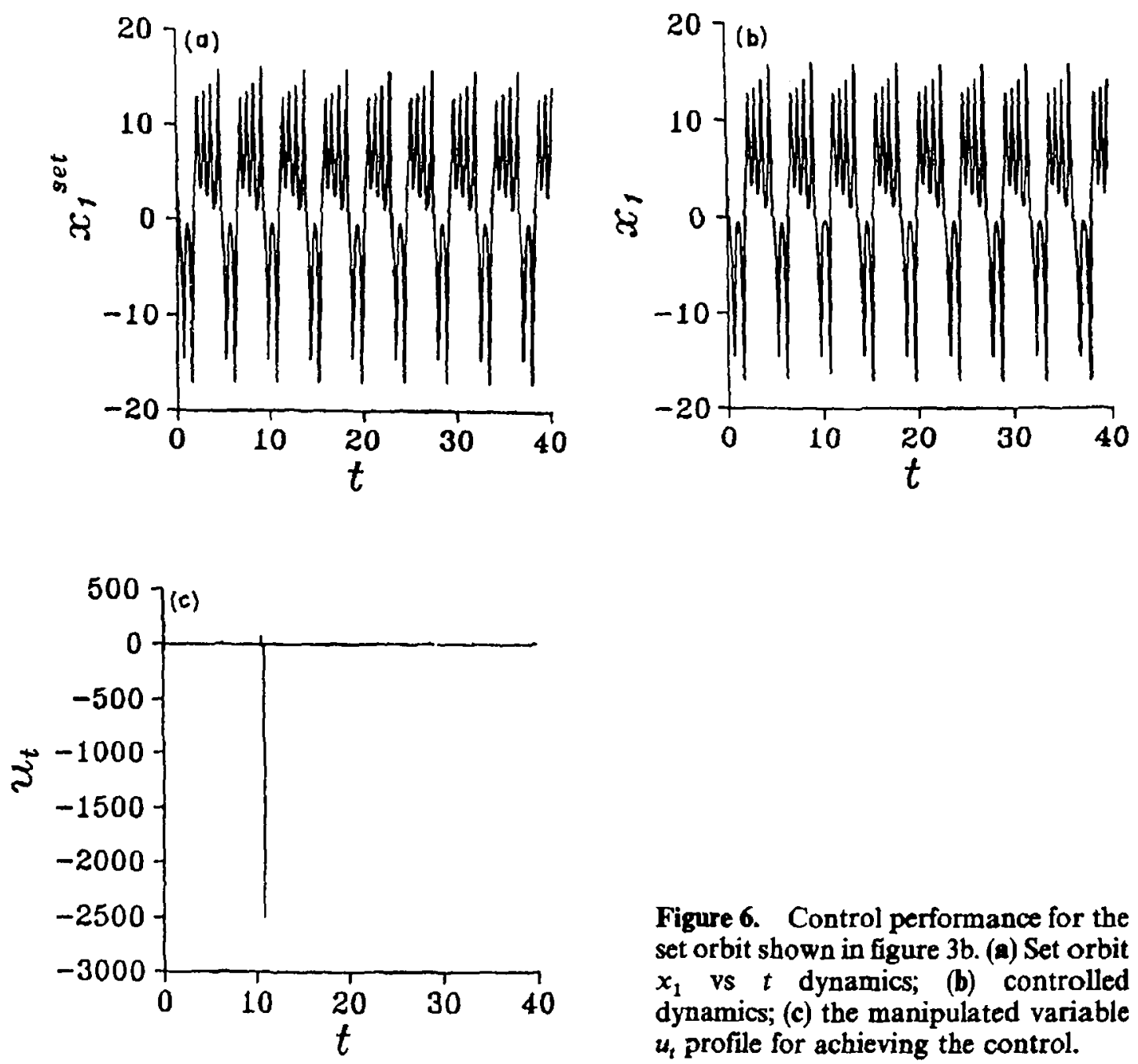

Figure 6. Control performance for the set orbit shown in figure 3 b. (a) Set orbit $x_{1}$ vs $t$ dynamics; (b) controlled dynamics; (c) the manipulated variable $u_{t}$ profile for achieving the control.

for each of the desired orbits are presented in figures $6 \mathrm{~b} \& 7 \mathrm{~b}$ with the manipulable variable profiles in figures $6 \mathrm{c}$ and $7 \mathrm{c}$. It is clearly evident from these figures that the controller is able to achieve the set trajectory with remarkable accuracy. However, it may be noted that the manipulated variable values (i.e. the Rayleigh number) to be implemented seem to be unconstrained and can assume large values. To avoid this problem we may choose an alternate manipulable parameter. Thus, choosing $b$ as $b+u_{t}$ in model (16), the design equation for the controller may be derived as

$$
\begin{aligned}
u_{t}= & {\left[\mathrm{r}\left(E_{1}+x_{1 s}\right)^{2}\left(F_{2}-E_{2}^{\prime}\right)\left(E_{1}+x_{1 s}\right)-E_{3}^{\prime}+F_{3}\right] /\left(E_{3}+x_{3 s}\right) } \\
& -b\left(E_{1}+x_{1 s}\right)^{2} .
\end{aligned}
$$

The controller movement to achieve the set trajectory (see figure 8a) is shown in figure $8 \mathrm{c}$ while figure $8 \mathrm{~b}$ represents the corresponding controlled profile. Note that the calculated values of the manipulable variable to be implemented are now realistic and vary between reasonable limits. This result suggests the need for a judicious choice of the parameter used for implementing control using IMC.

As a case study we now test the robustness of the algorithm for the Lorenz model in the presence of a noisy load disturbance for the controller design in (18). We have analysed a case study where $d_{1}$ is random in character with a Gaussian distribution having a mean of 0.01 and variance of 0.25 for all the three set-point orbits. For the 

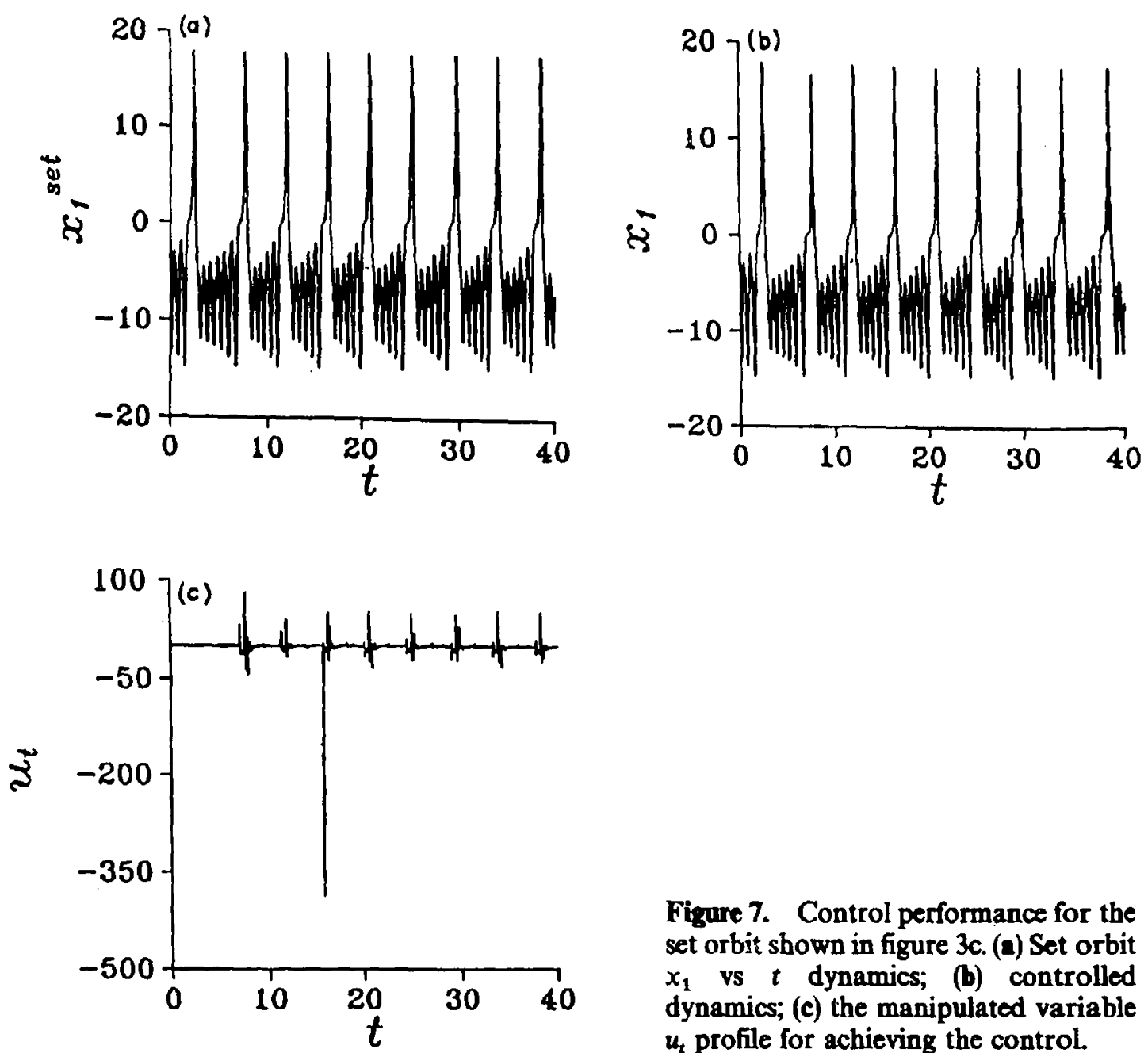

Figure 7. Control performance for the set orbit shown in figure $3 c$. (a) Set orbit $x_{1}$ vs $t$ dynamics; (b) controlled dynamics; (c) the manipulated variable $u_{t}$ profile for achieving the control.

first case, i.e. the set orbit in figure $4 \mathrm{a}$, the individual process outputs $x_{i}$ (iigures $5 \mathrm{~d}$, e \& f) exactly followed the desired process outputs $x_{i}^{\text {set }}$ (figures $5 \mathrm{a}, \mathrm{b} \& \mathrm{c}$ ), with the controller action shown in figure $5 \mathrm{~h}$. For the remaining two cases (i.e., for the set orbits in figures $6 \mathrm{a}$ and $7 \mathrm{a}$ ) control was again successful (see figures $9 \mathrm{a}$ and 10a, respectively) and indicated excellent disturbance rejection characteristics. It is interesting to note that the presence of noise considerably alters the magnitudes of manipulable variables to be implemented (compare figures $6 c$ and $9 b$ and $7 c$ and $10 b$ ).

Having shown the possibility of controlling chaos dynamically with the above studies for examples 1 and 2, we shall now turn our attention to controlling the autocatalator scheme. Using the general methodology we shall formulate a possible controller law for the system. However, for the sake of brevity we shall present the results of a simulation study where less successful control was achieved with a view to highlight the intricate nature of the control problem.

\subsection{Example 3: Control of the autocatalator scheme}

The three-variable autocatalator model capable of showing chemical chaos for reaction in a CSTR (Peng et al 1990, 1991) considers the presence of six reaction steps in the mechanism of the form

$$
\mathrm{P} \rightarrow \mathrm{A}, r_{1}=k_{0} p_{0},
$$



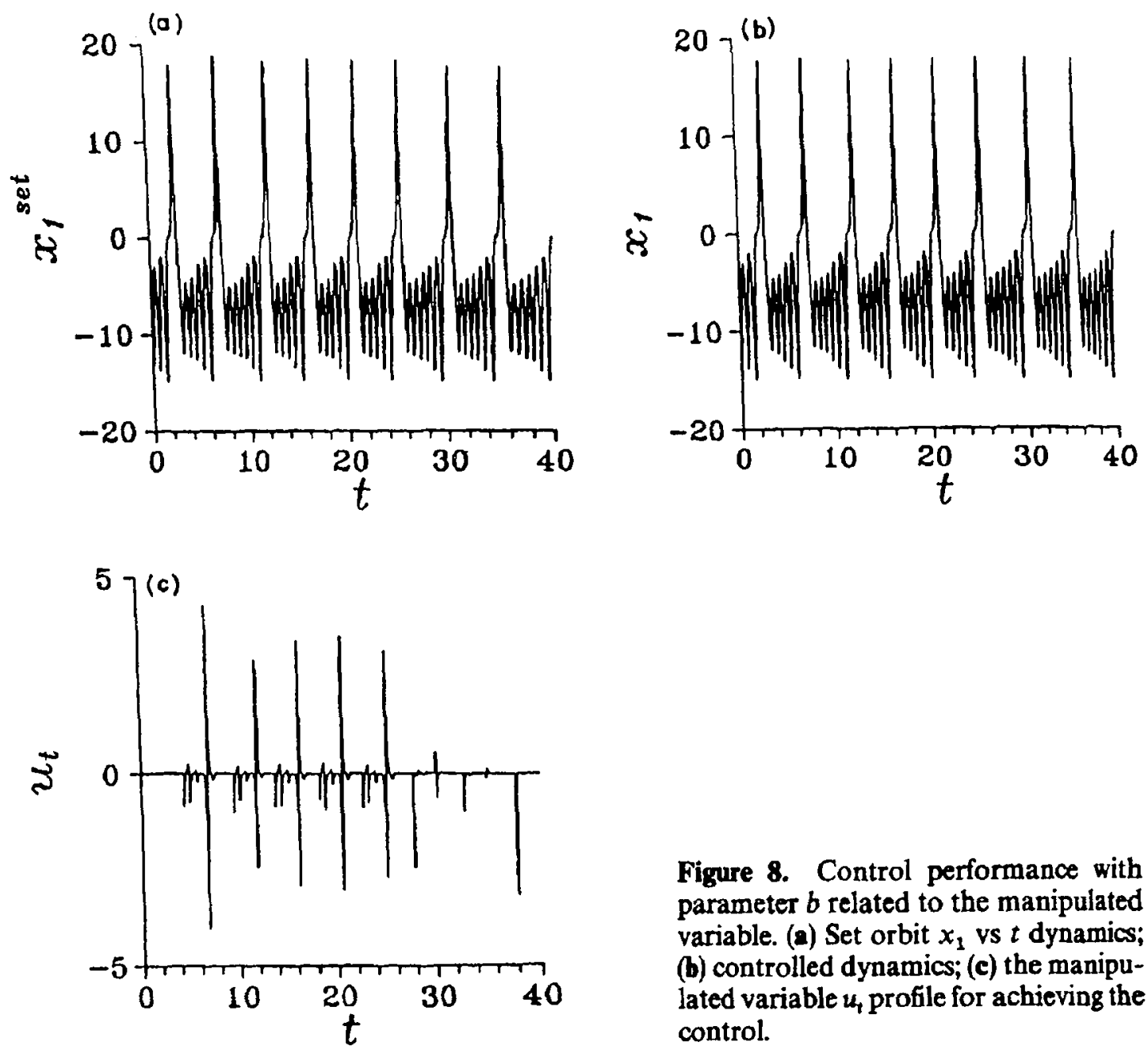

Figure 8. Control performance with parameter $b$ related to the manipulated variable. (a) Set orbit $x_{1}$ vs $t$ dynamics; (b) controlled dynamics; (c) the manipulated variable $u_{t}$ profile for achieving the control.
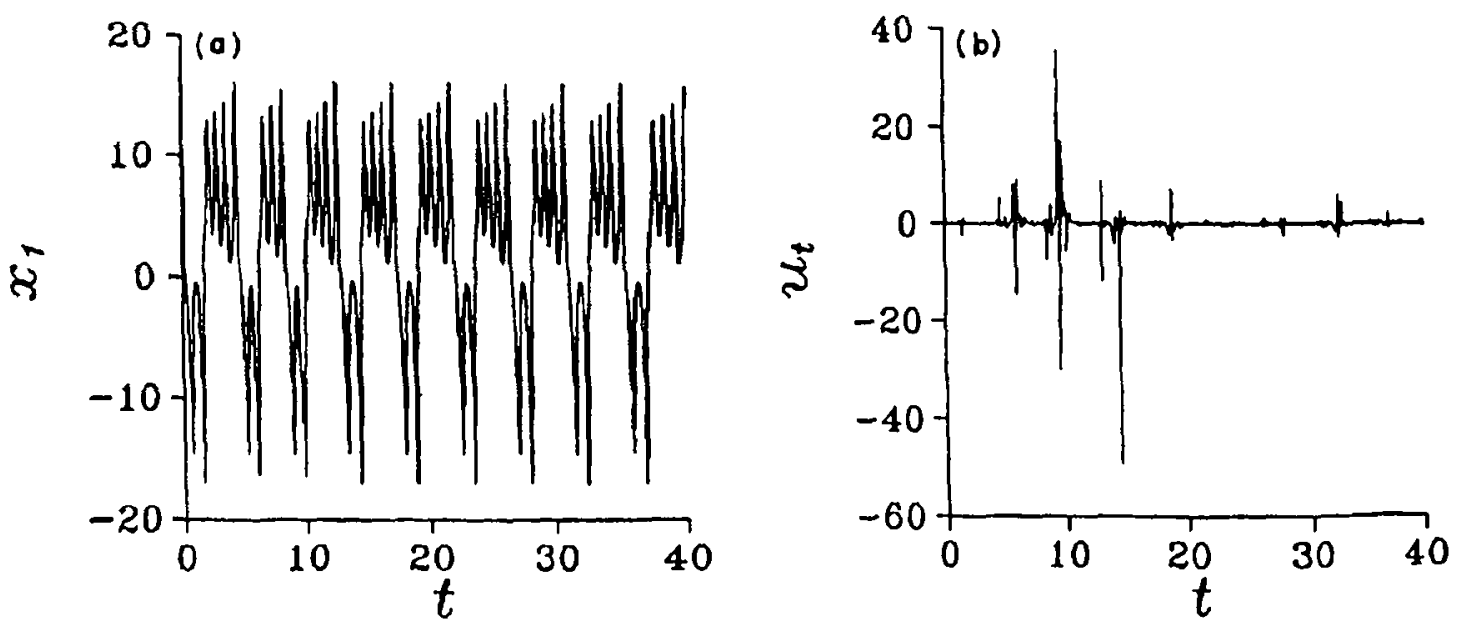

Figure 9. Control performance in the presence of stochastic load disturbance for a set orbit in figure 5a. (a) Controlled dynamics; (b) the manipulated variable $u_{t}$ profile for achieving the control. 

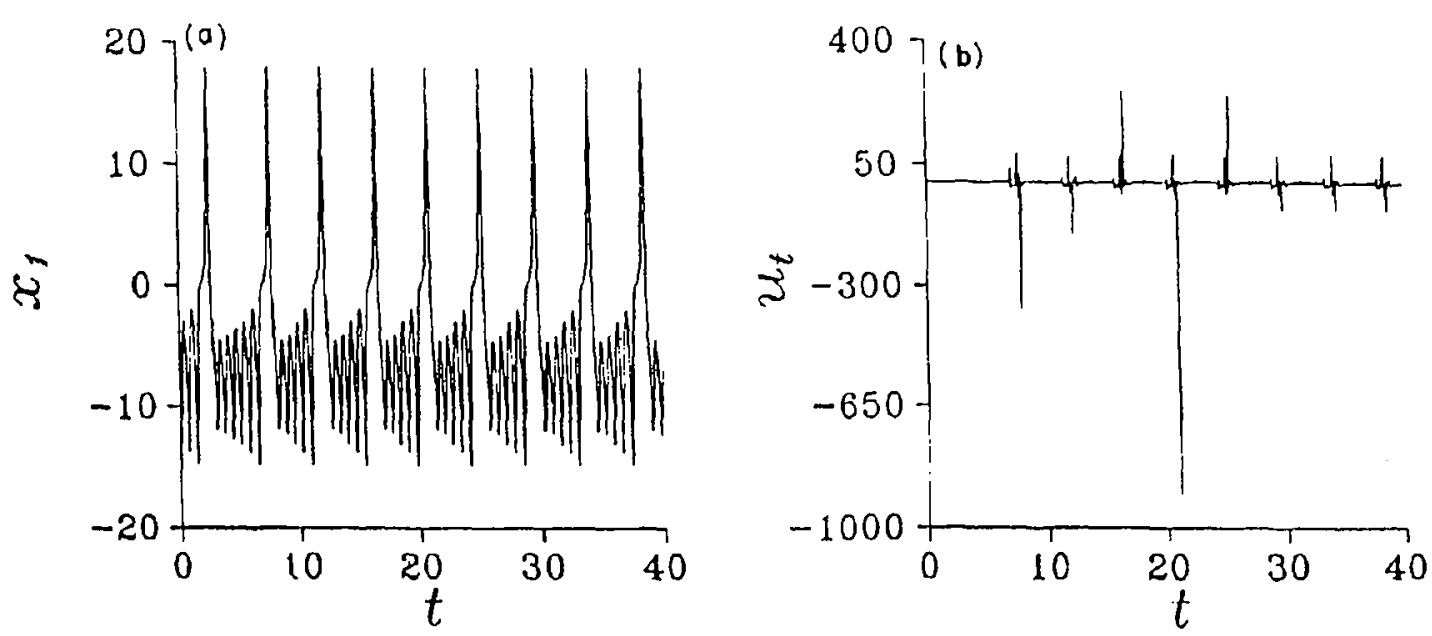

Figure 10. Control performance in the presence of stochastic load disturbance for a set orbit in figure 6a. (a) Controlled dynamics; (b) the manipulated variable $u_{t}$ profile for achieving the control.

$$
\begin{aligned}
& \mathrm{P}+\mathrm{C} \rightarrow \mathrm{A}+\mathrm{C}, r_{2}=k_{c} p_{0} c, \\
& \mathrm{~A} \rightarrow \mathrm{B}, r_{3}=k_{u} a, \\
& \mathrm{~A}+2 \mathrm{~B} \rightarrow 3 \mathrm{~B}, r_{4}=k_{1} a b^{2}, \\
& \mathrm{~B} \rightarrow \mathrm{C}, r_{5}=k_{2} b, \\
& \mathrm{C} \rightarrow \mathrm{D}, r_{6}=k_{3} c,
\end{aligned}
$$

where $r_{l}$ denotes the individual rate of the corresponding elementary step. The balance equations for the above scheme can be written in a compact dimensionless form in three variables (Peng et al 1990, 1991) as

$$
\begin{aligned}
& \mathrm{d} x_{1} / \mathrm{d} t=\left(\mu+u_{t}\right)\left(\kappa+x_{3}\right)-x_{1}-x_{1} x_{2}^{2}, \\
& \mathrm{~d} x_{2} / \mathrm{d} t=\left(x_{1}+x_{1} x_{2}^{2}-x_{2}\right) / \sigma, \\
& \mathrm{d} x_{3} / \mathrm{d} t=\left(x_{2}-x_{3}\right) / \delta,
\end{aligned}
$$

with the dimensionless variables and groups defined as

$$
\begin{aligned}
x_{1} & =\left(k_{1} k_{u} / k_{2}\right)^{1 / 2} a ; \quad x_{2}=\left(k_{1} / k_{u}\right)^{1 / 2} b ; \quad x_{3}=\left(k_{1} k_{3}^{2} / k_{u} k_{2}^{2}\right)^{1 / 2} c ; \\
\mu & =\left(k_{c} / k_{3}\right) p_{0} ; \quad \kappa=\left(k_{0} k_{3} / k_{c} k_{2}\right)\left(k_{1} / k_{u}\right)^{1 / 2} ; \quad \sigma=k_{u} / k_{2} ; \quad \delta=k_{u} / k_{3} ; \\
t & =k_{u} t^{\prime} .
\end{aligned}
$$

The modified model equations incorporating the local derivative deviations $\mathbf{F}$ is then,

$$
\begin{aligned}
& \mathrm{d} \hat{x}_{1, m} / \mathrm{d} t=\left(\mu+u_{t}\right)\left(\kappa+\hat{x}_{3, m}\right)-\hat{x}_{1, m}-\hat{x}_{1, m} \hat{x}_{2, m}^{2}+\theta F_{1}, \\
& \mathrm{~d} \hat{x}_{2, m} / \mathrm{d} t=\left(\hat{x}_{1, m}+\hat{x}_{1, m} \hat{x}_{2, m}^{2}-\hat{x}_{2, m}\right) / \sigma+\theta F_{2}, \\
& \mathrm{~d} \hat{x}_{3, m} / \mathrm{d} t=\left(\hat{x}_{2, m}-\hat{x}_{3, m}\right) / \delta+\theta F_{3,},
\end{aligned}
$$

and an expression for $u_{t}$ using the controller design procedure can be derived from (22) as

$$
u_{1}=\left\{\left[E_{1}^{\prime}+\sigma\left(E_{2}^{\prime}-F_{2}\right)+\left(E_{2}+x_{2 s}\right)-F_{1}\right] /\left[\kappa+\left(E_{3}+x_{3 s}\right)\right]\right\}-\mu .
$$




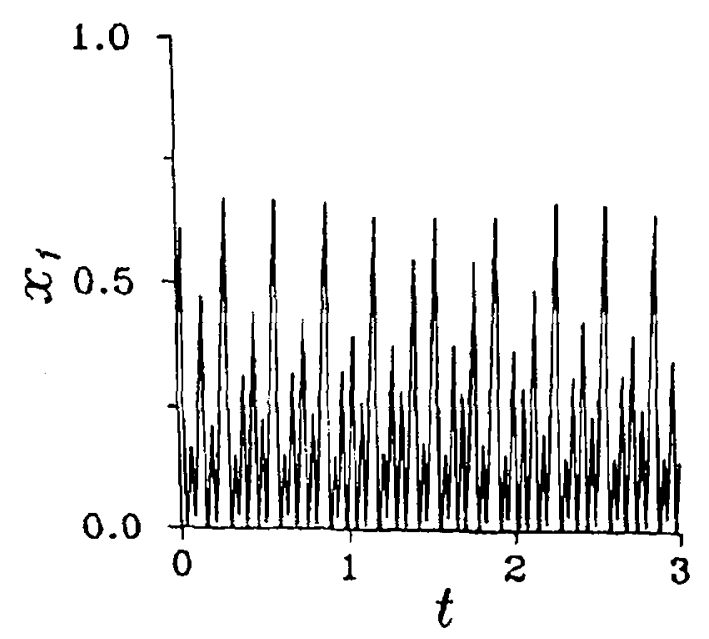

Figure 11. Open-loop $x_{1}$ dynamics for the autocatalator model.

For a set of process parameter values $\mu=0.154, \kappa=65.0, \sigma=5 \times 10^{-3}$, and $\delta=2 \times 10^{-2}$ the system shows chaotic behaviour (Peng et al 1991). Figure 11 shows the description in time of the process output $x_{1}$. Figure 12a shows the chosen system orbit along which we wish to control the system dynamics. With the controller switched on, the chaotic process was seen to intermittently follow the dynamics of the set orbit (as seen in figure 12b). Figure 12c shows the implemented values of the controller output $u_{1}$. The deviation from the set-point trajectory arises from the fact
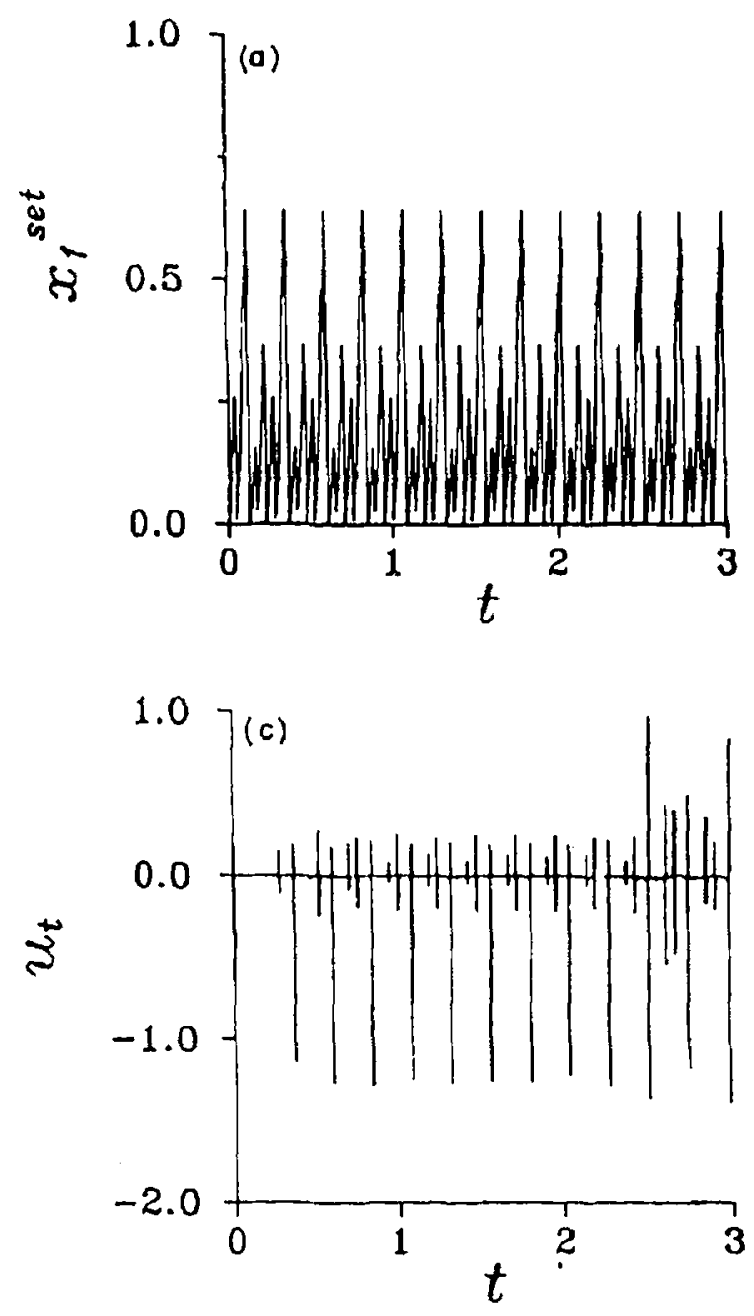

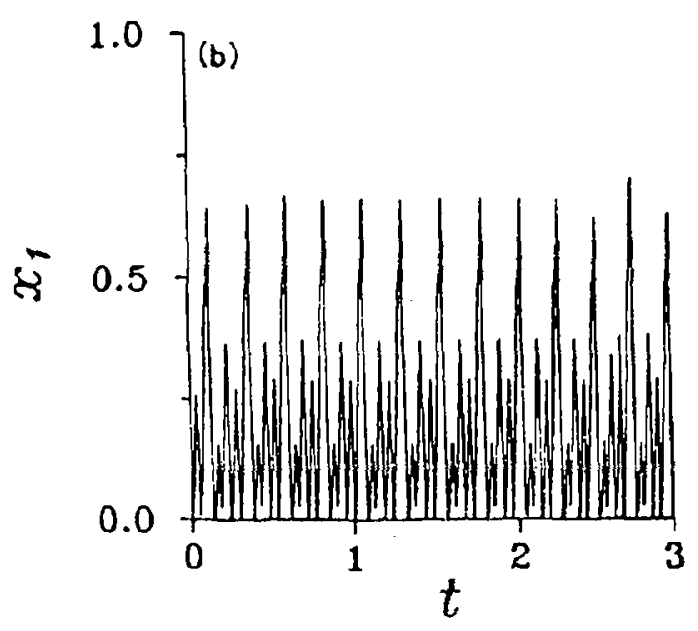

Figure 12. Control performance for the autocatalator model. (a) Chosen set orbit dynamics; (b) controlled dynamics; (c) the manipulated variable $u_{t}$ profile implemented during control. 
that divergent regions arise frequently in the phase space and the controller action cannot achieve the desired dynamics accurately enough. Some of the convergent regions were narrow to the extent that the stability properties altered during one step of integration. This difficulty causes errors in implementing the derivative corrections and distorts the error signals.

\section{Concluding remarks}

In this study, we have discussed and shown some of the intrinsic features of chaotic systems, which need to be taken into consideration for controlling their dynamics, and simulations bringing out these issues have been presented. The method used for controlling chaos show considerable promise, as exemplified by the results presented for the chaotic nonisothermal CSTR and the Lorenz models. The simulation study shown for the autocatalator indicates that the control efficiency depends both on the intrinsic nature of a nonlinear chaotic system and the stability properties of its dynamics.

\section{References}

Bai-Lin H 1984 Chaos (Singapore: World Scientific) pp. 54-61

Bandyopadhyay J K, Ravi Kumar V, Kulkami B D, Deshpande P B 1992 On dynamic control of chaos: A study with reference to a reacting system. Phys. Lett. A166: 197-204

Bandyopadhyay J K, Ravi Kumar V, Kulkarni B D 1993a Regulatory control of a chaotic nonisothermal CSTR. AIChE J. 39: 908-913

Bandyopadhyay J K, Ravi Kumar V, Kulkarni B D, Bhattacharya P 1993b Stabilizing the operation of a nonisothermal CSTR: A model study using a single variable nonlinear controller. Chem. Eng. Sci. 48: 3545-3553

Bequette B W 1991 Nonlinear control of chemical processes - A review. Ind. Eng. Chem. Res. 30: $1391-1413$

Berry M V, Percival I C, Weiss N O (eds) 1987 Dynamical chaos. Proc. R. Soc. London A413: $1-199$

Cutler C R, Ramaker B L 1980 Dynamic matrix control - a computer control algorithm. Proc. Joint Automatic Control Conf. (Philadelphia, PA: Instrum. Soc. Am.) Paper WP-5B

Ditto W L, Rauseo S N, Spano M L 1990 Experimental control of chaos. Phys. Rev. Lett. 65: $3211-3214$

Economou C G, Morari M, Palsson 1986 Internal model control 5. Extension to nonlinear systems. Ind. Eng. Chem., Process Des. Dev. 25: 403-411

Garcia C E, Morari M 1982 Internal model control 1. A unifying review and some new results. Ind. Eng. Chem., Process Des. Dev. 21: 308-315

Gattu G, Zafiriou E 1992 Nonlinear quadratic dynamic matrix control with state estimation. Ind. Eng. Chem. Res. 31: 1096-1.104

Grebogi C, Ott E, Yorke J A 1987 Chaos, strange attractors, and fractal basin boundaries in nonlinear dynamics. Science 238: 632-638

Guckenheimer J, Holmes P 1983 Nonlinear oscillations, dynamical systems and bifurcations of vector fields (New York: Springer Verlag)

Henson A M, Seborg D E 1991 An internal model control strategy for nonlinear systems. AIChE J. 37: 1065-1081

Hubler A 1989 Adaptive control of chaotic process. Helv. Phys. Acta 62: 343-346

Hubler A, Luscher E 1989 Resonant stimulation and control of nonlinear oscillations. Naturwissenschaften 76: 67-75

Isidori A 1985 Nonlinear control systems: An introduction (Berlin: Springer-Verlag)

Jackson E A 1990 The entrainment and migration controls of multiple-attractor systems. Phys. Lett. A151: 478-484 
Jackson E A, Hubler A 1990 Periodic entrainment of chaotic logistic map dynamics. Physica D44: $407-420$

Jorgensen D V, Aris R 1983 On the dynamics of a stirred tank with consecutive reactions. Chem. Eng. Sci. 38: 45-53

Jorgensen D V, Farr W W, Aris R 1984 More on the dynamics of a stirred tank with consecutive reactions. Chem. Eng. Sci. 39: 1741-1752

Kahlert C, Rössler O E, Varma A 1981 Chaos in a continuous stirred tank reactor with two consecutive first-order reactions, one exo-, one endothermic. Springer Ser. Chem. Phy's. 18: $355-365$

Kravaris C, Chung C 1987 Nonlinear state feedback synthesis by global input/output linearization. AIChE J. 33: 592-603

Kravaris C, Kantor J C 1990a Geometric methods for nonlinear process control 1 Background. Ind. Eng. Chem. Res. 29: 2295-2310

Kravaris C, Kantor J C 1990 b Geometric methods for nonlinear process control 2. Controller synthesis. Ind. Eng. Chem. Res. 29: 2310-2323

Kravaris C, Soroush M 1990 Synthesis of muitivariable nonlinear controllers by input/output linearization. AIChE J. 36: 249-261

Kulkarni B D, Tambe S S, Shukla N V, Deshpande P B 1991 Nonlinear pH control. Chem. Eng. Sci. 46: $995-1003$

Lee P L, Sullivan G K 1988 Generic model control. Comput. Chem. Eng. 12: 573-580

Limqueco L C, Kantor J C 1990 Nonlinear output feedback control of an exothermic reactor. Comput. Chem. Eng. 14: 427-437

Lorenz E N 1963 Deterministic nonperiodic flow. J. Atmos. Sci. 20: 130-141

Osledec V I 1968 A multiplicative ergodic theorem: Lyapunov characteristic numbers for dynamical systems. Trans. Moscow. Math. Soc. 19: 197

Ott E C, Grebogi C, Yorke J A 1990 Controlling chaos. Phys. Rev. Lett. 64: 1196-1199

Peng B, Petrov V, Showalter K 1991 Controlling chemical chaos. J. Phys. Chem. 95:4957-4959

Peng B, Scott S K, Showalter K 1990 Period doubling and chaos in a three-variable autocatalator. J. Phys. Chem. 94: 5243-5249

Ravi Kumar V, Kulkarni B D, Deshpande P B 1991 On the robust control of nonlinear systems. Proc. R. Soc. London A433: 711-722

Retzloff D G, Chan P C-H, Chicone C, Offin D, Mohamed R 1987 Chaotic behavior in the dynamical system of a continuous stirred tank reactor. Physica D25: 131-154

Richalet J, Rault A, Testud J L, Papon J 1982 Model predictive heuristic control applications to industrial processes. Automatica 14: 413

Roy R, Murphy T W Jr, Maier T D, Gills Z, Hunt E R 1992 Dynamical control of a chaotic laser: Experimental stabilization of a globally coupled system. Phys. Rev. Lett. 68: 1259-1262

Scott S K, Peng B, Tomlin A, Showalter K J 1991 Transient chaos in a closed chemical system. J. Phys. Chem. 94: 1134-1140

Shimada I, Nagashima T 1979 A numerical approach to ergodic problem of dissipative dynamical systems. Prog. Theor. Phys. 61: 1605-1616

Shukla N V, Deshpande P B, Ravi Kumar V, Kulkarni B D 1993 Enhancing the robustness of internal-model-based nonlinear $\mathrm{pH}$ controller. Chem. Eng. Sci. 48: 913-920

Sparrow C 1982 The Lorenz equations (New York: Springer-Verlag)

Thompson J M T, Stewart H B 1989 Nonlinear dynamics and chaos (New York: John Wiley \& Sons) 